\title{
Quantitative genome re-sequencing defines multiple mutations conferring chloroquine resistance in rodent malaria
}

\author{
Katarzyna Kinga Modrzynska ${ }^{1,8}$, Alison Creasey ${ }^{1}$, Laurence Loewe ${ }^{2}$, Timothee Cezard ${ }^{3}$, Sofia Trindade Borges ${ }^{4,9}$, \\ Axel Martinelli ${ }^{5}$, Louise Rodrigues ${ }^{5,10}$, Pedro Cravo ${ }^{5,11}$, Mark Blaxter ${ }^{3,6}$, Richard Carter ${ }^{1}$ and Paul Hunt ${ }^{1,7^{*}}$
}

\begin{abstract}
Background: Drug resistance in the malaria parasite Plasmodium falciparum severely compromises the treatment and control of malaria. A knowledge of the critical mutations conferring resistance to particular drugs is important in understanding modes of drug action and mechanisms of resistances. They are required to design better therapies and limit drug resistance.

A mutation in the gene (pfcrt) encoding a membrane transporter has been identified as a principal determinant of chloroquine resistance in $P$. falciparum, but we lack a full account of higher level chloroquine resistance. Furthermore, the determinants of resistance in the other major human malaria parasite, $P$. vivax, are not known. To address these questions, we investigated the genetic basis of chloroquine resistance in an isogenic lineage of rodent malaria parasite $P$. chabaudi in which high level resistance to chloroquine has been progressively selected under laboratory conditions.

Results: Loci containing the critical genes were mapped by Linkage Group Selection, using a genetic cross between the high-level chloroquine-resistant mutant and a genetically distinct sensitive strain. A novel high-resolution quantitative whole-genome re-sequencing approach was used to reveal three regions of selection on chr11, chr03 and chr02 that appear progressively at increasing drug doses on three chromosomes. Whole-genome sequencing of the chloroquine-resistant parent identified just four point mutations in different genes on these chromosomes. Three mutations are located at the foci of the selection valleys and are therefore predicted to confer different levels of chloroquine resistance. The critical mutation conferring the first level of chloroquine resistance is found in aat1, a putative aminoacid transporter.
\end{abstract}

Conclusions: Quantitative trait loci conferring selectable phenotypes, such as drug resistance, can be mapped directly using progressive genome-wide linkage group selection. Quantitative genome-wide short-read genome resequencing can be used to reveal these signatures of drug selection at high resolution. The identities of three genes (and mutations within them) conferring different levels of chloroquine resistance generate insights regarding the genetic architecture and mechanisms of resistance to chloroquine and other drugs. Importantly, their orthologues may now be evaluated for critical or accessory roles in chloroquine resistance in human malarias $P$. vivax and $P$. falciparum.

\section{Background}

Despite advances in vector control and attempts to develop effective vaccines, chemotherapy remains a principal mode of malaria control. Unfortunately malaria parasites resistant to drugs such as chloroquine (CQ) have arisen by gene mutation; their prevalence increasing

\footnotetext{
* Correspondence: Paul.Hunt@ed.ac.uk

'Institute for Immunology and Infection Research, University of Edinburgh, Edinburgh, UK

Full list of author information is available at the end of the article
}

by subsequent selection and transmission. These drug resistant parasites seriously compromise efforts to treat and control malarial disease both in individual cases and in communities. The ability to describe, understand and respond to these evolutionary processes continues to depend upon the identification of the precise genetic mutations which underlie the resistance phenotypes. Also, an identification of the genes involved may lead to insights regarding the mechanisms of drug action and

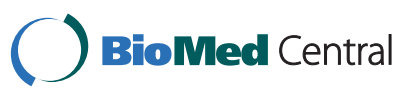


resistance, and the design of improved drugs and treatment strategies.

For chloroquine resistance (CQ-R), genetic linkage studies [1,2], other experimental approaches [3] and phenotype/genotype associations in parasites from natural infections [3-5] have mapped and identified the K76T mutation in the chloroquine resistance transporter, $p f C R T$, as the dominant genetic determinant in the most important human parasite Plasmodium falciparum. This protein mediates the export of CQ from the parasite digestive vacuole (DV) [6-8], its presumed site of action [9]. Additionally, specific point mutations in the multidrug resistance gene ( $p f m d r 1$ ) encoding an $\mathrm{ABC}$ transporter (P-glycoprotein homologue, $P g h-1$ ) have also been shown to modulate the level of resistance in CQ- R parasites in transfection experiments $[10,11]$ and in association studies using parasites from natural infections $[12,13]$. However, these two genes neither account for the full variation of in vitro $C Q$ responses, including high-level CQ-R (CQ-hiR) $[4,14]$ nor the appearance of CQ-R in another major human pathogen, $P$. vivax [15].

Understanding the genetic basis of CQ- $\mathrm{R}$ in the rodent malaria parasite, $P$. chabaudi could illuminate both of these questions; firstly, because, as in P. vivax, the orthologues of the $p f c r t$ and $p f m d r 1$ genes are not involved [16], at least in an existing lineage (Figure 1A) of parasites (strain AS); secondly because this same lineage contains parasites (e.g. AS-sens, AS-3CQ and AS-30CQ) with different levels of CQ-R $[17,18]$. Previous classical linkage analysis of a genetic cross between the CQ-R mutant AS$3 C Q$ and a genetically distinct sensitive strain, AJ, defined a region of $250 \mathrm{~kb}$ on chromosome 11 (chr11) as that containing the mutation conferring the first level of CQ-R $[19,20]$ but the critical gene or mutation was not identified. For P. chabaudi CQ-hiR in AS-30CQ, there has been no previous quantitative description of higher level CQ-R or CQ-hiR phenotypes, nor a systematic mapping of the genetic loci containing the critical mutations. Indeed, even the number of genes and mutations involved, and the sizes of their effect have remained undefined [18].

For drug resistance in malaria, classical linkage analysis of genetic crosses has proved effective in mapping single genes conferring clear phenotypes [1-3,21]. Also, Quantitative Trait Loci (QTL) approaches $[22,23]$ or genome wide association studies [24-26] have identified multiple major loci contributing to drug-resistance phenotypes. However, because these methods require the individual characterisation of many parasite lines or clones, they can be time-consuming or may fail to reveal the specific identity of a critical gene. Accordingly, Linkage Group Selection (LGS) [27] or QTL analysis were previously combined with Illumina ${ }^{\circledR}$ whole genome re-sequencing (WGS) to specify the mutations conferring resistance to artemisinin (V2728F ubp1) [28,29], sulphadoxine (K392Q $m d r 2$ ) [30], or mefloquine and lumefantrine resistance (mdr1 duplication) [31].

Here, we define the basic and high-level CQ-R phenotypes, extend the LGS strategy to map the multiple loci contributing to increasing levels of CQ-R, and use quantitative WGS of $>100,000$ single nucleotide polymorphisms (SNPs, differentiating the parental strains of the genetic crosses) to improve their resolution. We propose that, within these loci, mutations occur in the P. chabaudi ASlineage that confer CQ-R and CQ-hiR. We identify these and additional mutations using WGS.

\section{Results}

The $P$. chabaudi AS lineage contains parasites with increasing levels of CQ-R

In order to quantitate the CQ-R phenotypes in the AS lineage, the clones AS-sens, AS-3CQ and AS-30CQ $[17,18]$ (Figure 1A) were passaged in mice treated with either 0 , 3 or $10 \mathrm{mg} \mathrm{CQ} \mathrm{kg}^{-1}$ day $^{-1}$. The growth of these parasites (Figure 1B-D) demonstrated that there is an increasing level of resistance to CQ within the lineage. AS-sens parasites grew only in untreated animals. AS$3 \mathrm{CQ}$ grew at 0 and $3 \mathrm{mg} \mathrm{CQ} \mathrm{kg}{ }^{-1} \mathrm{day}^{-1}$ but not at $10 \mathrm{mg}$ CQ kg ${ }^{-1}$ day $^{-1}$ while AS-30CQ was able to survive $10 \mathrm{mg}$ CQ kg ${ }^{-1}$ day $^{-1}$. We therefore denoted the CQ responses of these clones as CQ sensitive (CQ-S), CQ-R or CQ-hiR, respectively. These data are consistent with a previous proposal that multiple mutations confer CQ-hiR [18] in this lineage, and suggest a suitable range of CQ doses for dissecting the critical genetic loci in LGS experiments, below. For example, we expected that parasites surviving $3 \mathrm{mg}$ CQ kg ${ }^{-1}$ day $^{-1}$ would be enriched with parasites having CQ-R (and, possibly, CQ-hiR) phenotypes, while those surviving $10 \mathrm{mg} \mathrm{CQ} \mathrm{kg}^{-1} \mathrm{day}^{-1}$ would be preferentially enriched with CQ-hiR parasites only.

\section{Improved LGS strategies resolve multiple large-effect genes}

In the case of drug resistance, LGS uses drug treatment to select the uncloned progeny of a genetic cross (between a drug-resistant clone and a genetically different drug-sensitive parasite) before measuring the proportions of parental alleles in the surviving parasites [32]. It generates a genome-wide scan of selection, revealing 'selection valleys' that are regions of the genome where the proportion of alleles from the drug-sensitive parent is greatly reduced (in drug-treated parasites relative to untreated parasites) and where the genes conferring resistance are located.

In the present study, an uncloned backcross (AS$30 \mathrm{CQ} \times \mathrm{AJ}$ ) between the CQ-hiR clone AS-30CQ and the genetically different $\mathrm{CQ}-\mathrm{S}$ parasite, $\mathrm{AJ}$ was generated and treated with different $C Q$ doses $(0,1.5,3,10$ or $20 \mathrm{CQ} \mathrm{kg}^{-1} \mathrm{day}^{-1}$, day 0-2 post-inoculation) to map 


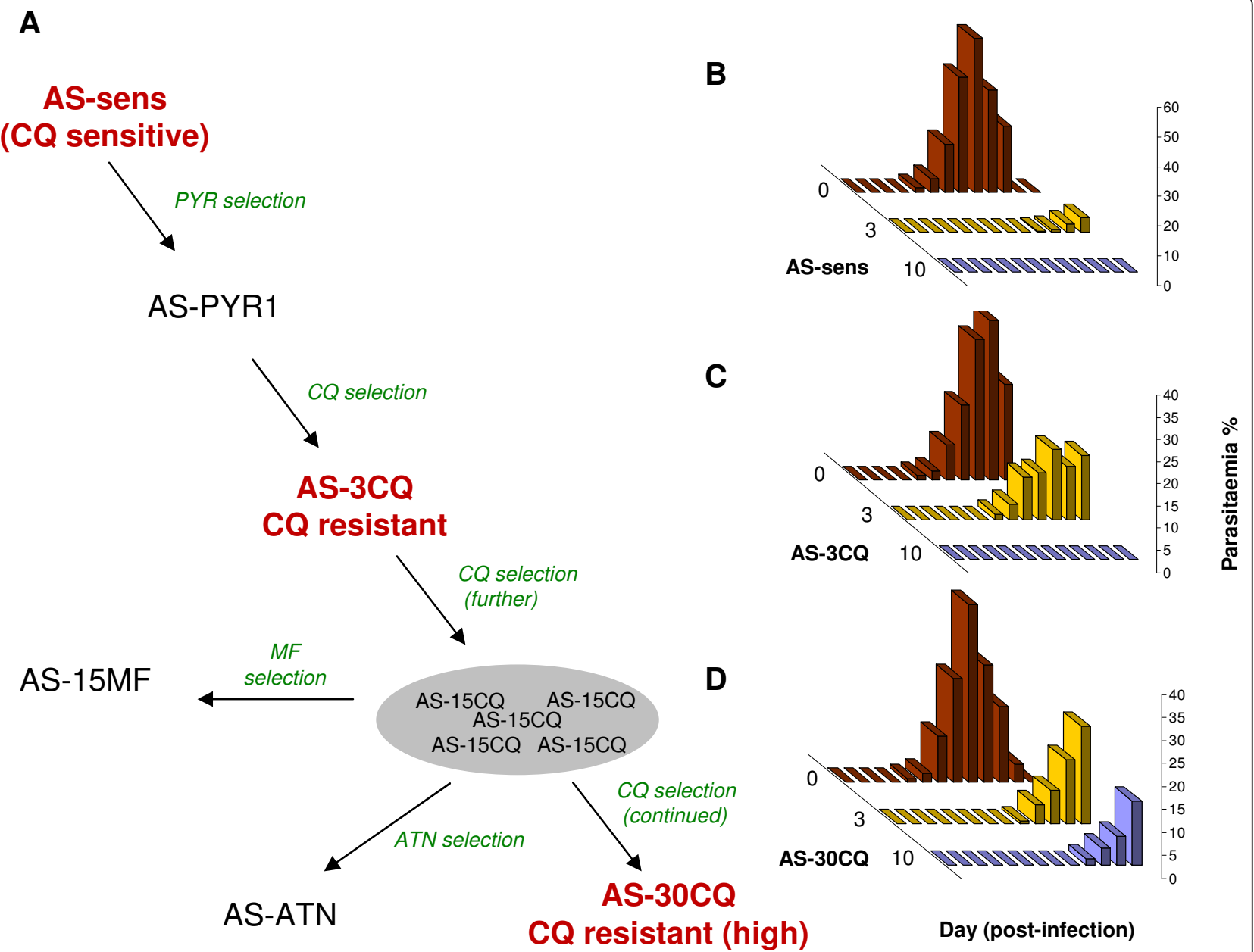

Figure 1 P. chabaudi clones in the AS lineage and their chloroquine responses. A. The AS lineage of drug resistant mutants $[35,55,56]$. Each arrow represents various regimes of selection using drugs (PYR, pyrimethamine; CQ, chloroquine; ATN, artesunate; MF, mefloquine). All strains

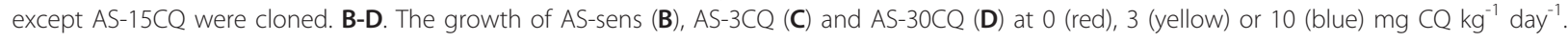

progressively the signatures of increasing CQ selection. Firstly, the proportions of parental alleles in all populations were measured in the surviving parasites, using a library of $\sim 96$ pyrosequencing assays [33] (LGS-pyro). Secondly, we developed a novel approach to improve the resolution and confidence of LGS mapping (see Methods, Additional File 1 (section 1)), thus. We defined an expanded set of genome-wide parental AS/AJ SNPs by WGS of the sensitive parent AJ (Additional File 2). 50-base paired-end reads (103-fold mean coverage) were mapped against the $18.8 \mathrm{Mb}$ Wellcome Trust Sanger Institute (Hinxton, Cambridge, UK) AS reference sequence (AS-WTSI). 92\% of the reads mapped uniquely. By filtering, we identified 104,667 high stringency SNPs in AJ relative to AS-WTSI at a mean frequency of $\sim 0.0056$ substitutions/nucleotide, similar to previous estimates of genetic diversity between the parental strains [34]. At these SNP positions, by counting short sequencing reads containing the AS or AJ base variant in populations of the LGS parasites surviving 0 or $3 \mathrm{mg} \mathrm{CQ} \mathrm{kg}^{-1} \mathrm{day}^{-1}$ (88-fold mean coverage for both), we quantitated the proportions of AS and AJ alleles, and investigated (for each SNP) the statistical significance of the difference between the allele proportion after each of the two treatments.

The proportions of alleles (genome-wide) in the LGS populations surviving $3 \mathrm{mg} \mathrm{CQ} \mathrm{kg}^{-1}$ day $^{-1}$, revealed by LGS-Illumina and by LGS-pyro were remarkably similar (Additional File 3) suggesting that the experimental errors incurred by either methodology were small.

LGS-pyro revealed progressively distinct selection valleys on chr11, chr03 and chr02 as the CQ dose increased (Figure 2A). LGS-Illumina confirmed selection valleys on chr11 (Figure 3A-D, Figure 4A) and chr03 (Figure 4A, B) at $3 \mathrm{mg} \mathrm{CQ} \mathrm{kg}{ }^{-1} \mathrm{day}^{-1}$. These data suggest that CQ-R phenotypes in the AS lineage are conferred by the action of a major effect gene on chr11, confirming previous linkage 
analysis $[19,20]$, and for CQ-hiR, major effect genes on chr03, and chr02.

\section{Mutation in aminoacid transporter (A173E aat1) is predicted to confer CQ-R}

LGS-pyro identified a partial selection valley on chr11 at $1.5 \mathrm{mg} \mathrm{CQ} \mathrm{kg}^{-1}$ day $^{-1}$ which proved to be dominant at $3 \mathrm{mg} \mathrm{CQ} \mathrm{kg}{ }^{-1}$ day $^{-1}$ (Figure 2A): For example, the proportion of an AJ allele of marker pcpf06-1338 decreased from $69.2 \%$ in untreated infections to $2.5 \%$ under CQ selection. Importantly, an independent genetic backcross between the mefloquine- and CQ-resistant clone AS-15MF [35] (in the same AS lineage, Figure 1A) and AJ also showed a similar distinct selection valley on chr11 at $3 \mathrm{mg} \mathrm{CQ} \mathrm{kg}^{-1}$ day $^{-1}$ (Figure $2 \mathrm{~B}$ ). Both selection valleys coincided with the $250 \mathrm{~kb}$ region previously mapped by classical linkage analysis [20] (Figure 2B).

LGS-Illumina confirmed the selection valley (at $3 \mathrm{mg}$ $\mathrm{CQ} \mathrm{kg}^{-1} \mathrm{day}^{-1}$ ) on chr11 at high resolution and statistical significance (Figure 3A-D). Here, a region at nucleotide $\sim 1,000,000$ where the proportion of AJ alleles reached a minimum $<3 \%$, was flanked on either side by regions of increasing AJ-allele proportion. The gradual and regular change in AJ allele proportion suggested the presence of many independent recombinant clones in the cross progeny. The differences in the gradients of the two slopes forming the selection valley may reflect different local recombination rates along the chromosome. These data resolved the presence of a mutation that confers CQ-R, close to nucleotide $1,000,000$ on chr11. This locus corresponds to the base of the selection valley as defined by LGS-pyro (Figure 2A, B) and to the $250 \mathrm{~kb}$ locus previously mapped [20], confirming that the gene bearing the mutation conferring CQ-R lies toward the right-hand end of chr11.

WGS (Methods, Additional File 1 (section 1)) identified a total of 7 point mutations (confirmed by dideoxysequencing) in AS-30CQ relative to AS-sens (Table 1, Table 2, Additional File 4), four of which are shared between AS-30CQ and AS-15MF [29]. Of these four mutations, only one maps to chr11; a non-synonymous mutation (A173E) in a gene (PCHAS_112780) encoding a predicted aminoacid transporter (aat1). It is found at base 996,332 (Sanger Sept2009 assembly) coincident with the floor of the chr11 selection valley (Figure $2 \mathrm{~B}, 3 \mathrm{~B}$ ). We concluded that the probability of failing to identify a genuine point mutation (false negative) in this region is very small, for three reasons. Firstly, $>\sim 96-98 \%$ of the AS-WTSI genome was covered by uniquely mapping short-reads (36 - $41 \mathrm{bp}$ ) employed here [29] (theoretical maximum $\sim 98.5 \%)$. Secondly, the read coverage is high: for $200 \mathrm{~kb}$ upstream and downstream of aat1 on chr11, only $0.61 \%$ or $0.73 \%$ of bases showed a read coverage of < 5 or $<10$, respectively (Additional File 5 ). Thirdly, we identified a very low overall genome-wide substitution frequency (7 point mutations/genome) in AS-30CQ (Table 1, Table 2) relative to AS-sens.

Dideoxysequencing confirmed that the A173E aat1 mutation first appeared in the AS lineage in AS-3CQ, along with the CQ-R phenotype (Table 1).

We therefore propose that aat1 A173E is the determinant of CQ-R in this particular $P$. chabaudi lineage.

The A173E aat1 mutation shares some properties with the determinant (K76T pfcrt) of CQ-R in P. falciparum. For example, like $p f c r t$, aat1 is predicted to encode a 10transmembrane (TM) helix transporter (Figure 5A) and its P. falciparum orthologue (PFF1430c) is targeted to the membrane of the DV (D. Fidock, P. Moura, pers comm.). The wild-type function of $p f c r t$ is uncertain but amino acid transport has been suggested [36,37]. Both K76T and A173E mutations result in negative charge shifts. Residue 173 in aat1 is at the start of a highly conserved region (Figure 5B) close to the start of the first TM-helix (TM1): in $p f C R T$, residue 76 lies at the start of TM1, predicted to be internal to the DV where CQ is thought to act. These data suggest that $A A T 1$ and $C R T$ may share some structure/function relationships impacting on their physiological function in the absence and/or presence of CQ.

\section{Mutation in another transporter (T719N PCHAS_031370) is predicted to confer intermediate CQ-R}

LGS-pyro experiments showed that AS markers on chr03 were selected at 3, 10 or 20 but not at $1.5 \mathrm{mg}$ CQ $\mathrm{kg}^{-1}$ day $^{-1}$ (Figure 2A). On chr03, the proportion of the AJ allele of marker pcpf02-0452 decreased from $79.3 \%$ (untreated) to $17.0 \%$ at $3 \mathrm{mg} \mathrm{CQ} \mathrm{kg}^{-1} \mathrm{day}^{-1}$. LGS-Illumina analysis confirmed that AJ alleles are reduced across the whole of chr03; from about $82 \%$ in untreated parasites to about $16 \%$ in CQ-treated population (Figure 4B). Details of the selection profile on chr03 are consistent with a focus of selection close to base $\sim 480,000$. The gradients of this selection valley are not dissimilar to those observed on chr11 when observed on a genome-wide scale (Figure 4A).

WGS of AS-30CQ revealed (Table 2) a non-synonymous mutation (T719N, PCHAS_031370) at base 474,123 on chr03, and dideoxysequencing confirmed that this mutation arose between AS-3CQ and AS-30CQ (Table 1,). However, this mutation does not appear in clones AS$15 \mathrm{MF}$ and AS-ATN (Figure 1A, Additional File 6) - these two clones being selected from AS-15CQ (non-clonal) using mefloquine and artesunate, respectively. Instead, AS-15MF and AS-ATN bear a 3 base deletion (I102del) in the same gene (confirmed by dideoxysequencing). We therefore suggest that both the T719N and I102del mutations were partially selected by intermediate levels of CQ in AS-15CQ prior to fixation during treatment with CQ (AS-30CQ), mefloquine (AS-15MF) or artesunate 

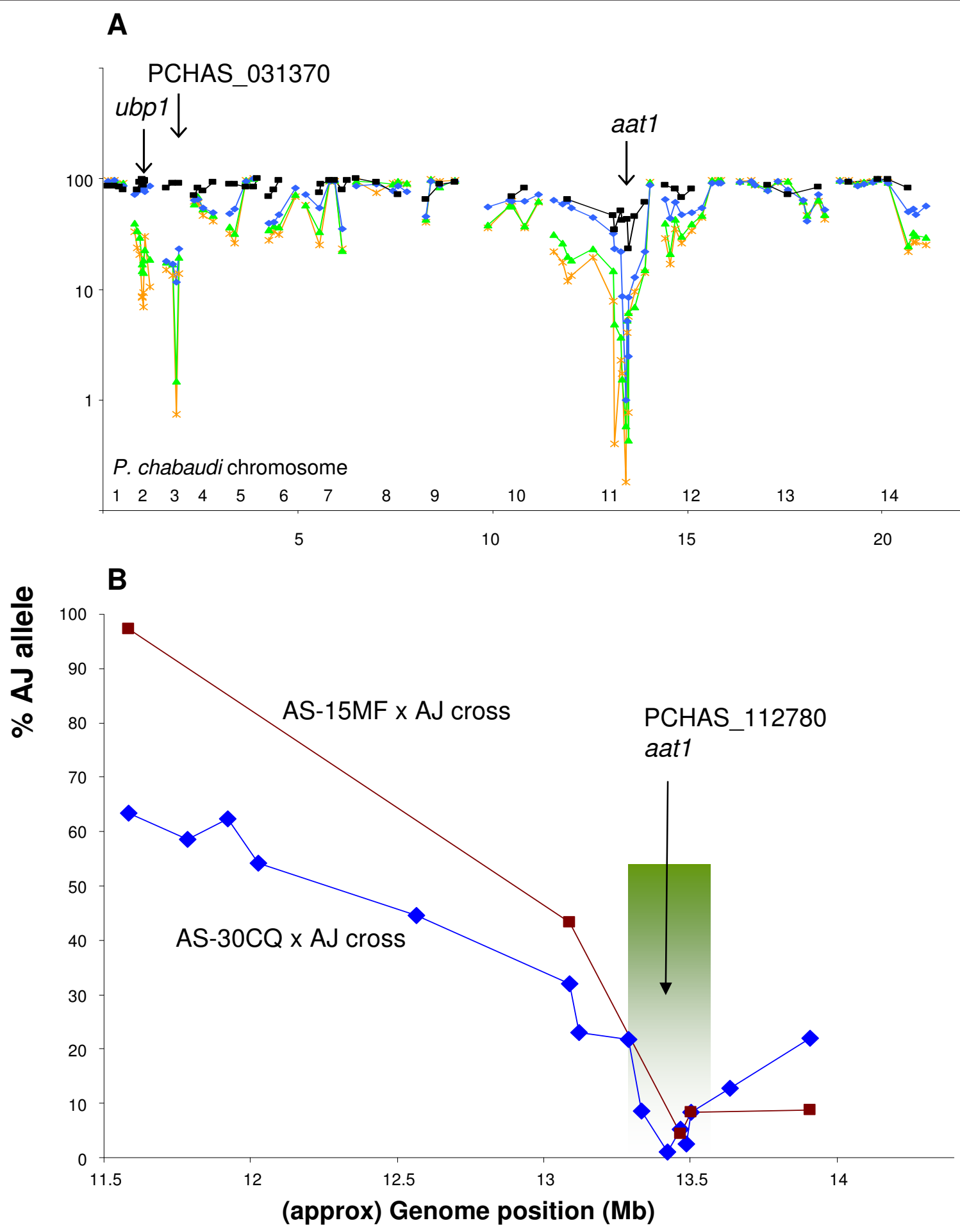

Figure 2 Scans of chloroquine selection (LGS-pyro). Allele proportions (sensitive strain, AJ) in uncloned progeny of genetic crosses using AS/ AJ SNPs (pyrosequencing). A. Genome-wide - AS-30CQ $\times$ AJ parasites surviving 1.5 (black, $\mathbf{- 1 )} 3$ (blue, $\$$ ), 10 (green, $\mathbf{4}$ ) or 20 (orange, $\boldsymbol{+}$ ) mg CQ $\mathrm{kg}^{-1}$ day $^{-1}$. The positions of mutations in aat1, PCHAS_031370 and ubp1 are indicated, and the proportions of the wild-type (AJ) base at these positions (as estimated by proportional sequencing [54]) are included. B. Chromosome 11 selection valley - parasites surviving $3 \mathrm{mg} \mathrm{CQ} \mathrm{kg}^{-1}$ day 1 , with position of aat1 mutation indicated; AS-30CQ $\times$ AJ backcross (blue, $\$$ ), AS-15MF $\times$ AJ backcross (red, $\mathbf{m})$. The region previously defined by classical genetic linkage analysis [20] is shown (gradient shaded green box). 


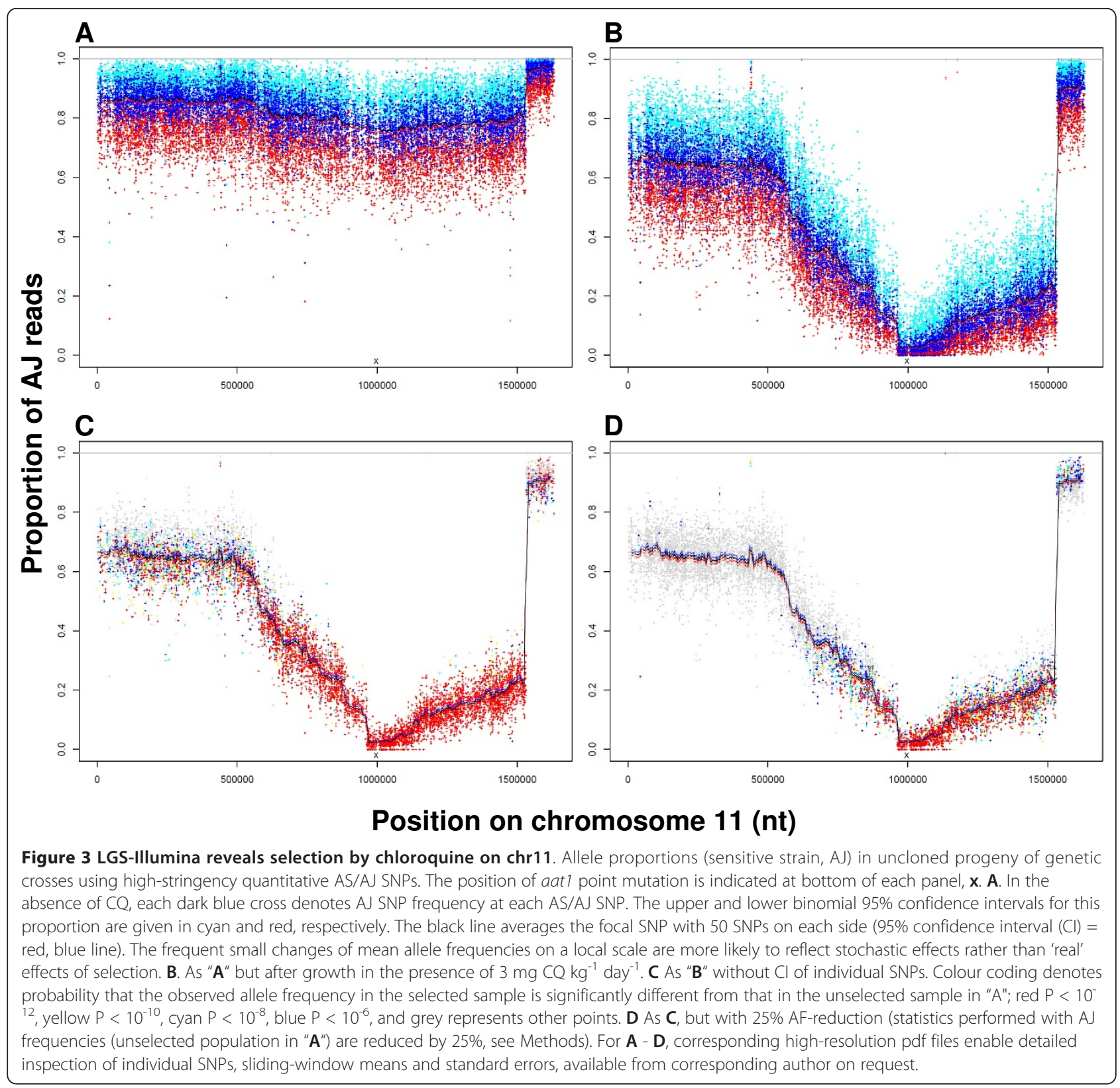

(AS-ATN) and subsequent cloning (Additional File 1, section 3).

PCHAS_031370 is predicted to encode a 12 TM-helix protein (Figure $5 \mathrm{C}$ ) and its $P$. falciparum orthologue PFB0675w is also predicted (but not yet experimentally confirmed) to target the DV membrane (D. Fidock, P. Moura, pers comm.). The T719N substitution occurs in a large loop between TM11 and TM12, a highly conserved region of the gene (Figure 5D). The I102del mutation is predicted to locate to the centre of TM3 and to change the overall prediction of TM domain structure (data not shown). The general transmembrane domain structure and DV localisation of PCHAS_031370 are therefore similar to other proteins ( $p f c r t, p f m d r 1$, aat1) identified as conferring or modulating CQ-R in P. falciparum or P. chabaudi. These data suggest that the PCHAS_031370 T719N mutation confers an increased CQ-R phenotype.

Interestingly, the P. yoelii orthologue (PY05194) of PCHAS_031370 lacks sequence corresponding to TM1 and TM2 that are otherwise present in other Plasmodium spp. (Figure 5D). Wild-type P. yoelii (17X) had not been exposed to antimalarial drugs but was reported to be highly CQ-R [38]. We suggest the possibility that P. yoelii CQ-R resistance might be related to this structural variation.

WGS of AS-30CQ also revealed a non-synonymous mutation T707N in PCHAS_030200 at base 70,553 on 


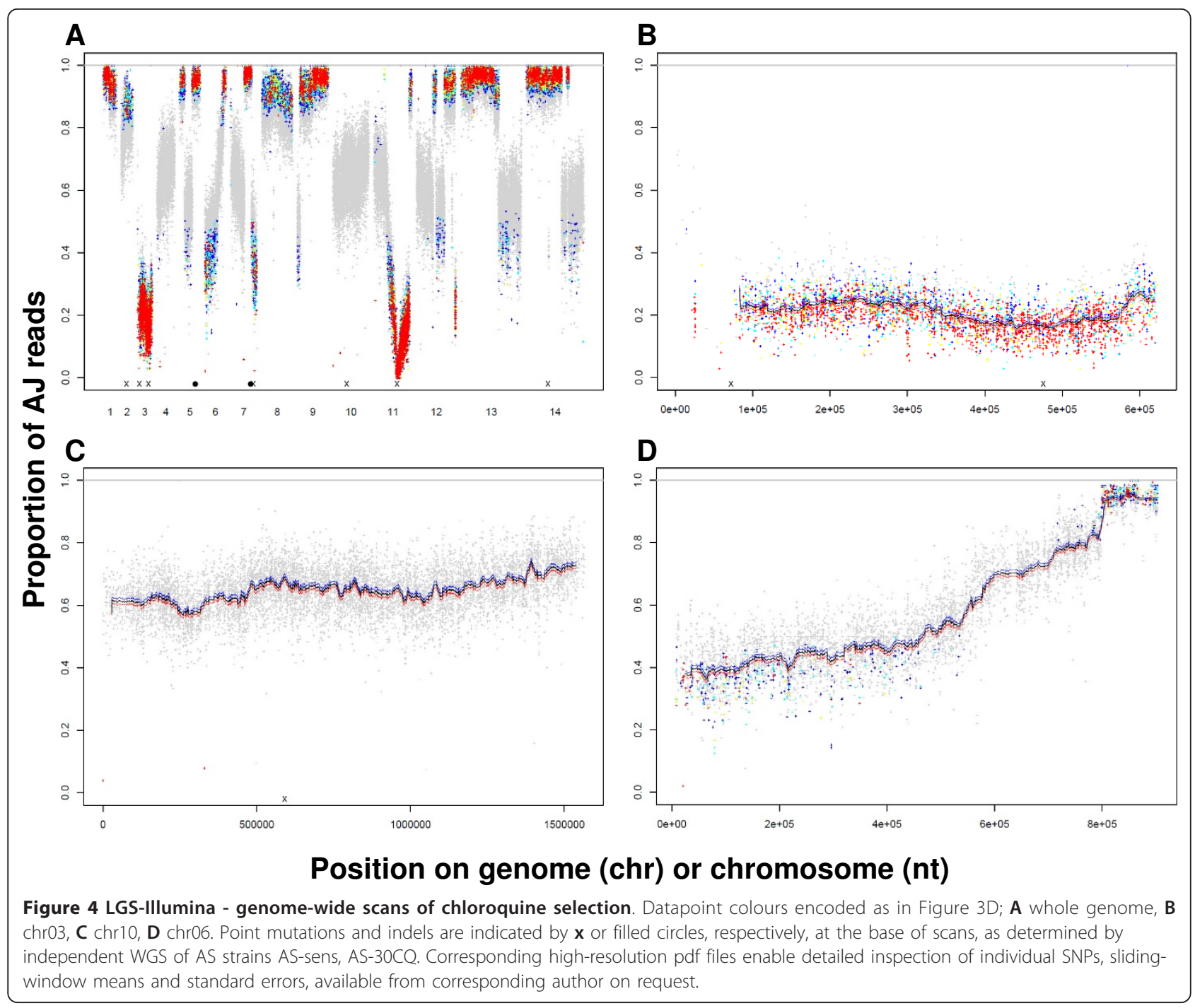

chr03 (Table 1, Table 2), predicted to encode a member of the $P$. chabaudi-specific variant gene family (chabaudi interspersed repeat, cir) [39]. This mutation maps to the left-hand of chr03. The detailed LGS-Illumina profile of AJ allele proportion (Figure 4B) supports the possibility that this mutation too may contribute to an augmented (intermediate) CQ-R. Dideoxysequencing confirmed that this mutation is specific to AS-30CQ and does not appear in either AS-15MF or AS-ATN.

\section{Mutation in de-ubiquitinating enzyme (V2728F Ubp1) is predicted to confer the highest level CQ-R}

LGS-pyro shows that AS markers on chr02 are selected at 10 and 20 , but not at $0,1.5$ or $3 \mathrm{mg} \mathrm{CQ} \mathrm{kg}^{-1} \mathrm{day}^{-1}$ (Figure 2A): e.g. at $10 \mathrm{mg} \mathrm{CQ} \mathrm{kg}^{-1} \mathrm{day}^{-1}$ on chr02, the percentage of AJ alleles of marker pcpf01-0158 decreased from $89.8 \%$ (untreated) to $14.0 \%$. Further resolution within the chromosome was not possible because parasites surviving 10 or $20 \mathrm{mg} \mathrm{CQ} \mathrm{kg-1}$ day $^{-1}$ were not analysed by LGS-Illumina. WGS had previously identified a single mutation (V2728F $u b p 1$, formerly V770F [28]) on chr02 [29] in both AS-30CQ and AS-15MF. It is the only mutation detected on chr02 and occurred between AS-3CQ and AS-15CQ (during CQ selection, Additional File 1 section 2). We conclude therefore that $u b p 1 \mathrm{~V} 2728 \mathrm{~F}$ confers CQ-hiR. This mutation was predicted to reduce the activity of a de-ubiquitinating enzyme [28] and also to confer artemisinin resistance in AS-30CQ, without previous exposure to this drug [29]. It is therefore predicted to affect the responses of malaria parasites to multiple drugs with diverse chemical structures and modes of action.

Dideoxysequencing confirms that this mutation appears in AS-30CQ and AS-15MF but not in AS-ATN. Instead an alternative mutation V2697F (formerly, V739F) $u b p 1$ appears in AS-ATN [28]. As with the 
Table 1 Phenotype and Genotype of Selected Clones of the AS P.chabaudi Lineage

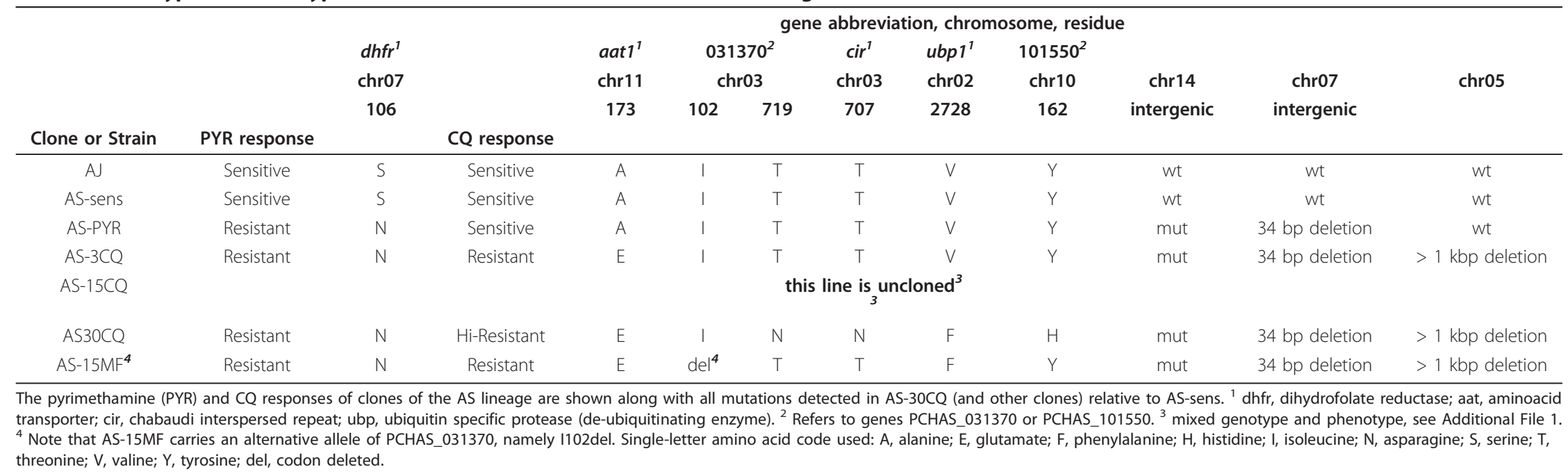


Table 2 Illumina ${ }^{\circledR}$ whole-genome re-sequencing - Confirmed and High-Confidence Mutations in AS-30CQ

\begin{tabular}{|c|c|c|c|c|c|c|c|c|c|}
\hline Chr & & Nucleotide number & WTSI & $\begin{array}{c}\text { AS- } \\
30 C Q\end{array}$ & $\begin{array}{l}\text { Quality } \\
\text { Score }\end{array}$ & Mutation & $\begin{array}{c}\text { Gene } \\
\text { (abbrev) }\end{array}$ & Nearest Gene & $\begin{array}{c}\text { P. falciparum } \\
\text { orthologue }\end{array}$ \\
\hline \multicolumn{10}{|c|}{ POINT MUTATIONS } \\
\hline 2 & & 216,954 & C & A & 99 & V2728F & PCHAS_020720 & & PFA0220w \\
\hline 3 & & 70,553 & G & $\mathrm{T}$ & 99 & $\mathrm{~T} 707 \mathrm{~N}$ & PCHAS_030200 & & none \\
\hline 3 & & 474,123 & C & A & 99 & T719 & PCHAS_031370 & & PFB0675w \\
\hline 7 & & 994,546 & G & A & 99 & $\mathrm{~S} 106 \mathrm{~N}$ & PCHAS_072830 & & PFD0830w \\
\hline 10 & & 634,932 & $\mathrm{~T}$ & C & 99 & $\mathrm{Y} 162 \mathrm{H}$ & PCHAS_101550 & & PF14_0279 \\
\hline 11 & & 996,332 & G & $\mathrm{T}$ & 99 & A173E & PCHAS_112780 & & PFF1430c \\
\hline 14 & & 936,945 & $\mathrm{~T}$ & G & 92 & & & PCHAS_142600 & PF08_0081 \\
\hline \multicolumn{10}{|c|}{ DELETIONS } \\
\hline & $\begin{array}{l}\text { Start (nucleotide } \\
\text { number) }\end{array}$ & $\begin{array}{l}\text { Finish (nucleotide } \\
\text { number) }\end{array}$ & & & & & & & \\
\hline 5 & 683,724 & 684,989 & & & & & PCHAS_051920 & & none \\
\hline 7 & 876,909 & 876,914 & & & & & & PCHAS_072420 & PF08_0067 \\
\hline
\end{tabular}

Summary of the high confidence mutations proposed in clone AS-30CQ (see Additional File 1). Read depth and quality scores fro mutations were according to SSAHA2. Start and Finish nucleotides for deletions are approximate only.

alternative mutations in PCHAS_011370, we suggest that these two alternative $u b p 1$ mutations are partially selected (by CQ treatment) in the uncloned parasite AS-15CQ. Their differential selection and fixation in clones AS30CQ, AS-15MF and AS-ATN derived from AS-15CQ after selection by $C Q$, mefloquine or artesunate are fully discussed along with a complete resolution of apparent contradictions regarding their linkage (or otherwise) with alternative mutations in PCHAS_031370 (Additional File 1, section 3).

\section{Other mutations in AS-30CQ}

Nine mutations are identified in AS-30CQ relative to ASsens; seven point mutations and two deletions (Table 1 , Table 2).

Four point mutations (on chr11, chr03 (two) and chr02) are associated with signatures of CQ selection and were discussed above. They all first appeared in the P. chabaudi AS lineage (Figure 1A) in AS-3CQ or AS-30CQ (i.e. during CQ selection).

A fifth point mutation was identified in AS-30CQ, as predicted, on chr07. This mutation, S106N dhfr (encoding dihydrofolate reductase) was confirmed by dideoxysequencing to have first appeared in AS-PYR1. It was previously shown to confer resistance to pyrimethamine $[30,32,40]$.

Four mutations (two point mutations and two deletions), identified in AS-30CQ, were not associated with signatures of drug-selection. Three were confirmed by dideoxysequencing; a non-coding point mutation on chr14, a $34 \mathrm{bp}$ non-coding deletion on chr07 (Additional File 7) (both first appearing in AS-PYR1) and a non-synonymous point mutation on chr10, namely Y162H PCHAS_101550 (orthologue of P. falciparum PF14_0279) arising first in AS30CQ (i.e. during CQ selection). A fourth mutation could not be confirmed by dideoxysequencing: extensive low or zero-coverage and/or a small cluster of poor quality SNP calls in AS-30CQ, (also in AS-15MF [29] but not in AS$50 \mathrm{~S} / \mathrm{P}$ [30] strongly suggested a $\sim 1 \mathrm{~kb}$ deletion on chr05 occurring first in AS-3CQ or AS-15CQ (i.e. during CQ selection, Figure 1A). Other studies will be required to evaluate whether these 2 point mutations and 2 deletions are consistently neutral (and consequently randomly fixed during cloning), or whether they play a minor role in drug (pyrimethamine or CQ) resistance. Such roles could include a weak selective advantage in the presence of drugs or compensation for fitness costs incurred by the 'drug resistance' mutations (for example, in the absence of drugs or during transmission of parasites through mosquitoes).

The low probability of failing to identify point mutations (false negatives) on chr11 was discussed in the AAT1 section above. Similar arguments and data may be applied equally to the whole genome (Additional File 5) and are addressed more fully here (Additional File 1) and previously [29]. Our conclusion is that the probability of a false negative point mutation in central regions of a chromosome is low $(<0.05)$. For regions of chromosomes closer to the telomeres where $P$. chabaudi-specific genes are located, we suggest that the probability of a false negative is higher but not easily quantified. However, with the exception of possible selection at the left hand end of chr06, our experiments show no evidence of CQ selection in these regions.

\section{Genome-wide scan of selection - other observations}

Both LGS-pyro and LGS-Illumina data indicated that AJ allele proportions were high $(\sim 90 \%)$ in the untreated LGS population but were reduced after drug treatment (Figure 2A, 4A) at many loci genome-wide, including chromosomes other than chr02, chr03 or chr11. For example across chr10 (Figure 4C) AJ allele proportions 


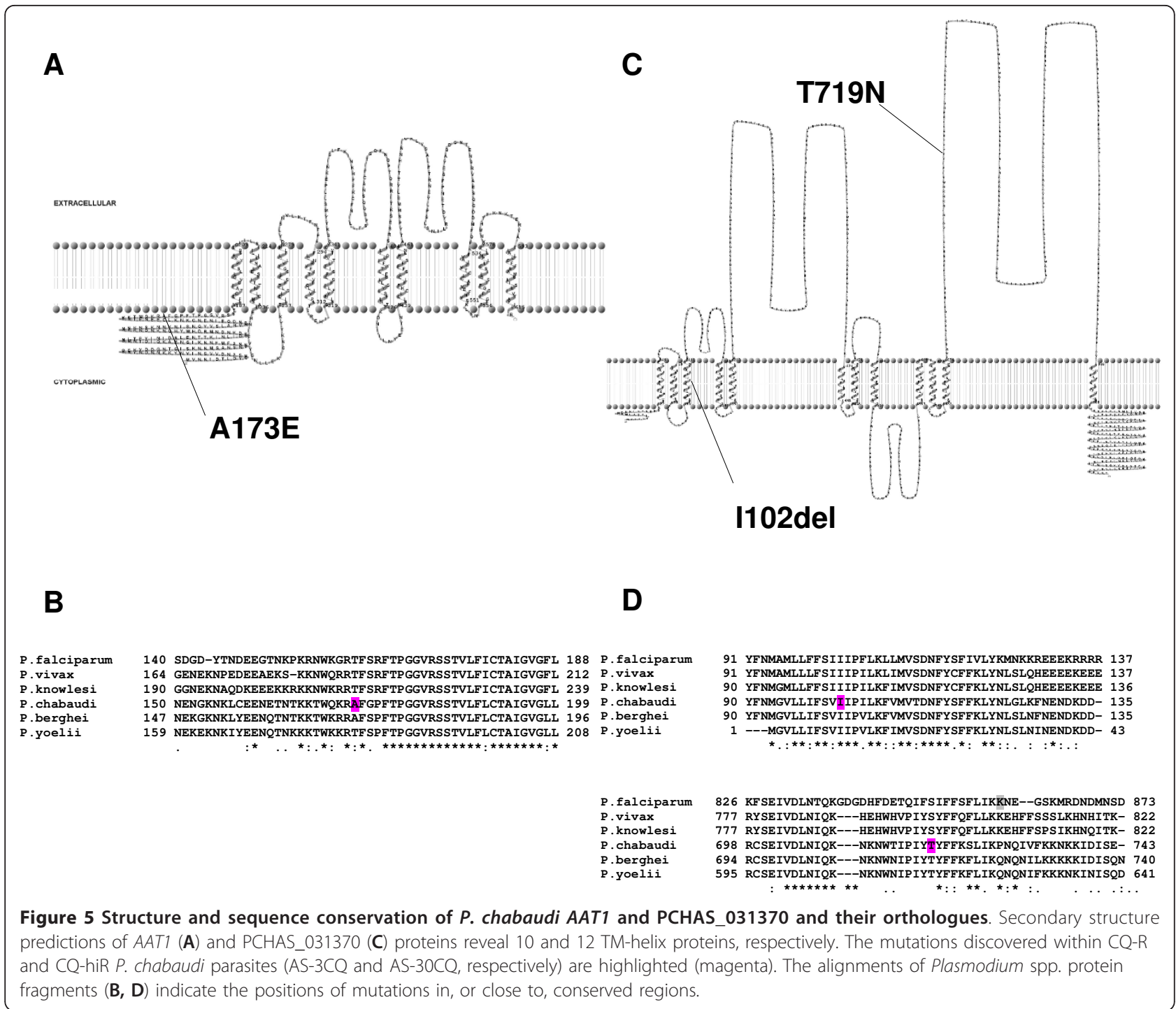

were reduced from $\sim 86 \%$ to $\sim 65 \%$, after drug treatment (3 mg CQ $\mathrm{kg}^{-1} \mathrm{day}^{-1}$ ). These data may reflect high AJ proportions in the backcross and the loss of AJ parental parasites (present in a significant proportion) after CQ treatment. Additionally, or alternatively, AJ alleles may have been positively selected during growth without drugs, reflecting the possible action of multiple (small effect) genes that underlie the faster growth of AJ compared to AS parasites, observed routinely in previous experiments [41,42].

The LGS-pyro data showed that the selection valleys on chr11, 03 and 02 were produced progressively at increasing CQ doses (Figure 2A). Thus, low doses resulted in the selection of AS alleles on chr11, and increasing doses resulted in selection of AS alleles on chr03 and then on chr02. We note that the maximum depth of the chr11 selection valley was reached at a lower CQ dose than that required to achieve maximum selection at chr03 (and additionally for chr02). These data may be interpreted by invoking two possible factors. Firstly, we suggest that the mutations conferring CQ-hiR on chr03 and on chr02 may incur 'fitness costs': i. e. that in the absence of a sufficiently high concentration of $C Q$, these mutations may reduce the growth of parasites. This would mean that, at lower CQ concentrations, parasites with CQ-R (bearing only the $173 \mathrm{E}$ aat 1 allele) would be selected to a greater degree than CQ-hiR parasites bearing multiple mutations. Secondly, the effects of the mutations on chr03 and/or chr02 may be epistatic to the A173E aat1 mutation, because mutated AS alleles at these loci (chr03 and chr02) only show signs of selection (at higher doses of CQ) after the selection of mutated AS alleles at the aat1 locus (chr11) (at lower doses of CQ). According to this interpretation, parasites bearing only the mutations on 
chr03 or chr02 (or both) are not selectable by lower doses of CQ.

The LGS-Illumina analysis revealed an abrupt discontinuity of AJ proportion at the right hand end of chr11 (Figure 3A-D) and similar changes on chr05, 07, 09, 12, 13 and 14 (Figure 4A, Additional File 8), often in both untreated and drug-treated parasites. These are described and discussed in Additional File 1, section 4. These discontinuities are also observable in the LGS-pyro data. Our conclusion is therefore that they are not artefacts of LGS- Illumina. Also, they did not arise by natural genetic or selection phenomena. They are most likely to arise from differences (in genome assembly) between ASWTSI (reference strain) and our parental strains AS-sens and/or AJ.

LGS-pyro and LGS-Illumina revealed regions showing possible weak drug selection but where mutations were not detected; for example, the left-hand end of chr06 (Figure 4D). Further studies are required to investigate whether these represent reproducible regions of selection or arise from random variation.

\section{Discussion}

We have described in vivo CQ-R and CQ-hiR phenotypes in the genetically related $P$. chabaudi AS parasites AS$3 C Q$ and $A S-30 C Q$, mapped the underlying genetic loci on chr11 (CQ-R), chr03 and chr02 (CQ-hiR) and, by WGS, have identified a small number of mutations proposed to confer these phenotypes. A173E aat1 (on chr11) is proposed to confer CQ-R. T719N PCHAS_031370 (transporter on chr03) and V2728F $u b p 1$ (on chr02) are proposed to confer CQ-hiR. We also suggest that T707N PCHAS_030200 (cir gene on chr03) may also contribute to CQ-hiR.

\section{Mutations conferring CQ-R are identified by integrating genetic and genomic data}

The genetic (LGS-pyro and LGS-Illumina) and genomic (WGS) approaches employed showed a remarkable concordance on chr02, chr03 and chr11: i.e. these major selection valleys contain 4 of the 6 mutations that arose in the lineage under CQ selection (from AS-PYR1 to AS-30CQ). The genome-wide scans do not reveal selection around the other two mutations arising during CQ selection (Y162H PCHAS_101550, chr10; > 1 kb deletion, chr05) nor around those (three) arising during previous pyrimethamine selection (in AS-PYR1, Figure 1A); i.e. 34 bp deletion and S106N dhfr) (both chr07), non-coding point mutation (chr14).

The correspondences between the genetic and genomic data and the arguments deployed previously [29] and above (Results - other mutations in AS-30CQ) together suggest that only 3-4 major effect genes conferring CQ-R and CQ-hiR were fixed by strong selection (and cloning) during experimental evolution from AS-sens to AS-30CQ. Furthermore, only a small number of weakly selected or effectively neutral mutations were fixed stochastically during population bottlenecks such as those occurring during transmission of parasite lines, or experimental cloning.

Previous similar investigations in the $P$. chabaudi AS lineage demonstrated that one mutation conferred resistance to each of pyrimethamine, sulphadoxine [30], mefloquine [31] and artemisinin [29] and that $\leq 3$ mutations were fixed stochastically each time during the selection and cloning of AS-50S/P, AS-15MF and AS-30CQ respectively. The low rate of nucleotide substitution, even during multiple passages and bottlenecks, makes this system well adapted for investigations of resistance to other drugs.

\section{The genetic architecture of CQ- $R$}

CQ-hiR was previously generated in AS-30CQ of the P. chabaudi AS-lineage by drug selection over about 40 passages $[17,18]$, leading to the suggestion that the CQhiR phenotype arose by a series of small steps and was mediated by several mutations at different loci, although the number of mutations was not predicted. Actually, for CQ-R, the present data strongly support the contribution of aat1 (chr11) as a single major effect gene, confirming previous classical genetic linkage analysis $[19,20]$. For CQ-hiR, two (or three) major effect genes (PCHAS_031370 (transporter) and $u b p 1$, on chr03 and chr02, respectively) are strongly supported. In this respect, the architecture of CQ- $\mathrm{R}$ in $P$. chabaudi may be similar to that of $P$. falciparum where $p f c r t$ and $p f m d r 1$ alleles appear to generate high levels of CQ resistance epistatically $[12,13]$. The hypothesis that CQ-hiR in $P$. chabaudi is mediated by a large number of smalleffect mutations is rejected by the data presented here.

\section{The genetic determinants of CQ-R}

The concordance between the phenotypic, genetic and genomic data strongly supports the identification of mutations in $A A T 1$ as the key determinant of CQ-R. We have also revealed shared properties of pfcrt and aat 1 and their mutations (K76T, A173E, respectively), such as putative function (amino acid transport), subcellular location, TMhelix topology, type and position of mutation. Genomewide transcription profiling of the CQ-S P. falciparum parasite (strain 106/1) and CQ-R transfectants identified the aat 1 orthologue PFF1430 as one of a small number of differentially expressed genes [37] which also included genes predicted to encode transporters, proteases and components of the trafficking pathways. This suggests future transfection and functional studies to address questions regarding the role of these mutations in amino acid or CQ transport and CQ-R in P. falciparum and P. chabaudi. The involvement of aat1, another (chr03 encoded) DV transporter and $u b p 1$ in CQ-hiR also provoke 
questions regarding the relationship between haemoglobin digestion, protein turnover, amino acid transport/availability and CQ action, export and resistance phenotypes.,

Interestingly, the V2728F $u b p 1$ mutation has now been shown to confer resistance to both artemisinin [29] and chloroquine (this study). This resolves a previously unexplained result - that artemisinin resistance mediated by mutations in $u b p 1$ appeared before parasites were exposed to artemisinin. This data also suggests that there must be some commonality in the molecular mechanisms of resistance to the two drugs, at least in the P. chabaudi AS lineage.

\section{The rodent model and public health}

P. chabaudi and P. falciparum have similar or identical genetic bases for resistance to pyrimethamine (point mutations in $d h f r$, [30]) and to mefloquine ( $m d r 1$ amplification) [31]. For CQ-R, both parasite species share key features (e.g. reduced CQ accumulation in DV [43], changes in DV morphology [44] and verapamil reversibility [45]). Now, notwithstanding the evidence supporting the proposal that different genes confer CQ-R in the two species, the data presented here suggest that some features of the molecular mechanism of resistance acquisition may be similar. This supports the use of the rodent model to identify candidate genetic markers of resistance to future antimalarial drugs. Furthermore, the orthologues of aat1 (PVX_114575, PFF1430c) and PCHAS_031370 (PVX_002795, PFB0675w) should now be evaluated as candidate molecular markers of CQ-R in P. vivax and modulators of CQ responses in P. falciparum.

The experiments reported here share some features previously exploited by genome-wide selection scans in yeast [46], genome-wide association [26] and high-resolution identification of mutated genes $[47,48]$ in P. falciparum. The present study demonstrates how similar approaches can be used to identify genes conferring complex selectable phenotypes such as drug-resistance in experimental systems.

\section{Conclusions}

Previously, increasing chloroquine resistance phenotypes were experimentally selected in a lineage of the rodent malaria, Plasmodium chabaudi. Here, these phenotypes were measured, the underlying genetic loci mapped and mutations specified using a novel quantitative genetics and genomics approach.

This approach analysed genetic crosses by selecting progeny en masse at different drug concentrations. The frequencies of parental alleles in the surviving parasites were measured using $\sim 100$ pyrosequencing single nucleotide polymorphism (SNP) assays, and for $\sim 100,000$ single nucleotide polymorphisms, by Illumina short-read sequencing. This defined 'selection valleys' on chromosomes 11,
3 and 2, where genes conferring resistance were expected to be located. Whole genome re-sequencing of the chloroquine resistant mutant parasite and the sensitive progenitor wild-type parasite showed that only 7 point mutations in the whole genome had arisen in the lineage. The specific isolated mutations within the selection valleys were identified. A mutation in a putative aminoacid transporter (aat1) encoded on chr11 confers chloroquine resistance. Mutations in another transporter (PCHAS_031370, chr03) and a deubiquitinating enzyme ( $u b p 1$, chr02) confer higher level chloroquine resistance. Orthologues of these genes in $P$. falciparum and $P$. vivax can now be studied for their contribution to chloroquine resistance in human infection. These data will generate insights of the mechanism of chloroquine resistance in human and rodent malaria parasites.

\section{Methods}

\section{Parasite and mouse strains, routine passage}

$\mathrm{AJ}$ and AS-sens are chloroquine (CQ) sensitive clones of the rodent malaria Plasmodium chabaudi chabaudi, isolated from wild-caught Thamnomys rutilans thicket rats from the Central African Republic [49]. AS-PYR, AS-3CQ and AS-30CQ were selected (and cloned) sequentially from AS-sens by pyrimethamine, CQ and high CQ concentrations progressively and respectively [18,50]. All resistant phenotypes were heritable and stable after cloning, freeze/thaw cycles, passage of parasite without drug, and after transmission through the mosquito host. Six- to eight-week old laboratory CBA female mice were used for all the experiments with the exception of mosquito transmission when C57/BL6 mice were used. All animals were housed and maintained according to the standard animal husbandry conditions, with free access to food (RM3 diet) and PABA supplemented water. All experiments were conducted in compliance with the United Kingdom Animals (Scientific Procedures) Act 1986.

\section{Drug phenotyping}

Mouse infections were initiated with an intraperitoneal inoculum of $10^{6}$ parasites and treated with CQ sulphate (Beacon Pharmaceuticals) diluted to appropriate concentration with water and administered to a $20 \mathrm{~g}$ mouse by gavage in $100 \mu \mathrm{l}$. The treatment was repeated for the first 3 days of infections ( $\mathrm{d} 0-2$ post-infection (p.i.)). The parasitaemia of all infections was monitored by daily thin blood smears as described previously [29]. Each treatment group consisted of three animals.

\section{AS-30CQ $\times$ AJ backcross and LGS}

The AS-30CQ $\times$ AJ cross was performed by allowing Anopheles stephensi mosquitoes to feed upon anaesthetised C57/BL6 mice infected with mixture of both strains, according to the protocol established previously [29]. 
After 14-15 days, salivary glands were dissected and sporozoites injected into donor mice. The recovered crossprogeny asexual forms were further passaged, treated with $0,1.5$ or $10 \mathrm{mg} \mathrm{CQ} \mathrm{kg}{ }^{-1}$ day $^{-1}$ for three days, and pooled in order to increase the numbers of resistant recombinants in the mixture. The resulting parasites were backcrossed with the sensitive parent (AJ) using the same procedure as above. The backcross recombinant progeny were selected en masse with $0,1.5,3,10$ and 20 mg CQ kg ${ }^{-1}$ day $^{-1}$ (d0-2 p.i.) in groups of 5 mice each. When parasitaemias within a group reached $>10 \%$, blood was harvested, pooled and DNA isolated using the protocol previously described [34].

\section{Pyrosequencing}

The PSQ ${ }^{\mathrm{TM}}$ HS-96A pyrosequencing system was used to measure the proportion of $\mathrm{AJ}$ alleles in all selected backcross populations. A set of $\sim 96$ uniformly spaced, quantitative pyrosequencing assays measuring the proportion of the SNPs between the AS and AJ were designed as previously described [33]. The pyrosequencing assays were prepared and performed according to the manufacturer's instructions, each assay being performed in triplicate, on three different template samples.

\section{Genome re-sequencing and mutation detection in AS-30CQ}

The chloroquine resistant AS-30CQ genome and that of its sensitive progenitor AS-sens were sequenced with 50 and 36 base single reads at approx 80- and 40-fold coverage, respectively by the GenePool Genomics Facility http://genepool.bio.ed.ac.uk/. The single end reads obtained for AS-30CQ were aligned against the isogenic $P$. chabaudi AS strain reference genome ftp://ftp.sanger.ac. uk/pub/pathogens/P_chabaudi/Archive/September_2009_assembly/ using the MAQ [51]http://maq.sourceforge.net/ maq-manpage.shtml and SSAHA2 [52] ftp://ftp.sanger.ac. uk/pub/zn1/ssaha_pileup/ssaha_pileup-readme software suites. The SNPs and indels between the two strains were identified and analysed (Additional File 1, section 1) as previously described [29].

\section{Quantitative sequencing (LGS-Illumina)}

$\mathrm{AJ}$ and the AS-30CQ $\times \mathrm{AJ}$ backcross (surviving 0 or $3 \mathrm{mg} \mathrm{CQ} \mathrm{kg}^{-1}$ day $^{-1}$ ) were each sequenced using $\sim 2 \mu \mathrm{g}$ of DNA on single lanes, using 50 base paired end reads that were mapped against the AS-WTSI reference sequence (PlasmoDB 6.3 version, 17 Feb 2010) using BWA (v0.5.8) software [53]. For AJ/AS SNP detection, unique reads with mapping quality $>30$ and bases with base quality (Phred-like code) > 20 were used. SNPs were called in positions covered by at least 10 reads if at least $30 \%$ of the bases are different from the reference.

\section{SNP selection}

113,746 candidate AS/AJ SNPs were determined by Illumina ${ }^{\circledR}$ whole-genome re-sequencing (WGS) of CQ-sensitive parental strain AJ (relative to reference strain sequence AS-WTSI). Of these 838 (0.74\%) are in contigs that are currently not assembled in the 14 chromosomes and were ignored in the analysis presented here. For inclusion in the plots presented in this work, the remaining 112,908 SNPs were further filtered using the following criteria: (i) $\geq 20$ reads in all samples (i.e. the AJ sequence sample, the untreated cross-progeny sequence sample and the CQ-treated cross-progeny sequence sample, (ii) $>90 \%$ frequency of the AJ basecall in the AJ sequence sample (usually this is 100\%). SNPs failing these criteria $(7,079)$ were excluded leaving 104,667 SNPs for the genome wide scan of selection. The numbers of AS and AJ nucleotide calls at these SNPs were determined from the sequencing data of AJ and AS-30CQ $\times$ AJ (untreated and treated at $3 \mathrm{mg} C Q$ $\mathrm{kg}^{-1}$ day $^{-1}$ ) samples using custom scripts.

\section{Statistics - Binomial test}

The quantitative LGS-Illumina analysis samples reads from a population of individual clones that are the result of the recombination and selection processes described above. For each SNP the finite sampling size results in random deviations from the expected value for the true allele proportion. These random deviations can be described by the binomial distribution, which is the basis of the tests for statistical significance we employ. As expectation $p$ we use the point estimate obtained from our data, where the number of successes' $x$ are given by the number of AJ alleles observed and the total number of attempts $n$ is given by the sum of $\mathrm{AJ}+\mathrm{AS}$ alleles sequenced (usually equal to the number of reads containing the SNP). We use a two-sided binomial test with a confidence limit of $95 \%$ throughout the paper. This means that strong AFreductions (see below) can result in "significant" points at high $\mathrm{AJ}$ allele frequencies, indicating possible selection for AJ alleles.

\section{Statistics - sliding window analysis}

Because the frequencies of individual SNPs come with binomial variability, we sought to estimate the local AJ proportions with reduced sampling error by combining neighbouring SNPs in a sliding window analysis (on the assumption that the real AJ allele frequency was constant over this scale). We summed $x$ and $n$ for 101 neighbouring SNPs (50 on each side of a focal site) and computed upper and lower limits (using binomial test described above), shown by blue and red lines in Figure 3A-D and Figure 4A-D. 


\section{Statistics - AF-reduction}

As described in 'Results' and elsewhere [29], there are factors that may reduce $\mathrm{AJ}$ allele proportions at loci not linked to the drug resistant phenotype in drug-treated samples relative to untreated samples. The effects of these factors are very difficult to estimate precisely. We therefore increased the stringency of our statistical analysis by applying a correction parameter, termed "Allele Frequency Reduction" (AF-reduction). This parameter reduces the observed \% of AJ alleles in the untreated population (in order to correct for additional and confounding factors, such as the removal of AJ parental parasites). To test the robustness of our conclusions we tested the significance of differences between the drug-treated and the untreated samples with AF-reduction $=0 \%$ and $25 \%$. We then computed the probability that our drug-treated samples could have been produced by allele frequencies as observed in the point estimates of our untreated samples. These probabilities are indicated by the colour of each SNP shown (Figure 3C, D and Figure 4A-D). In these plots, we highlight probability $\mathrm{P}<10^{-6}$ as an important threshold by coloured data points. The choice of this threshold can be justified by considering the large number of SNPs used in this test (approximate Bonferroni correction). At AFreduction $=25 \%$ we found 7342 SNPs that deviated from their expected values with $\mathrm{P}<10^{-12}$ (red in Figure 3C, D, 4A-D), 3588 with $10^{-12}<\mathrm{P}<10^{-10}$ (yellow), 5945 with $10^{-}$ ${ }^{10}<\mathrm{P}<10^{-8}$ (cyan) and 9272 with $10^{-8}<\mathrm{P} 10^{-6}$ (blue). In these SNPs, the AJ-allele proportion was either below or above the expected values with the specified probability. Lower SNP frequencies indicate the valleys of selection caused by resistance mutations. Higher SNP frequencies could indicate the presence of $\mathrm{AJ}$ alleles benefiting the growth of parasites in the presence of drugs and other more complex growth, virulence and immunity traits. Alternatively, many significant higher SNP proportions might indicate that the AF-reduction operation was too large.

\section{The confirmation of predicted mutations}

The presence of all mutations predicted between the ASsens and AS-30CQ clones was confirmed using standard PCR and dideoxysequencing using primers presented in Additional File 9. Additional clones from the AS lineage (AS-PYR, AS-3CQ, AS-15MF, AS-ART and AS-ATN) were also tested, when required, to determine and confirm when mutations arose in the lineage.

\section{Proportional sequencing}

The proportions of mutant alleles at aat1, PCHAS_031370 and $u b p 1$ genes were measured in untreated and treated populations of parasites using proportional sequencing as described previously [54]. The fragments containing the pre-defined mutations were amplified and sequenced using specific primers (Additional File 9). The resulting electropherograms were analyzed using Chromas 2.33 software (Technelysium Pty Ltd) and the heights of peaks corresponding to the wild-type and mutated nucleotides measured. These were used to calculate an index of the respective proportion of the wild-type allele (AJ) in the population.

\section{Homology studies and protein structure predictions}

The orthologues of mutated genes were identified in other Plasmodium spp. using PlasmoDB database and the alignments produced using ClustalW2 software http://www.ebi.ac.uk/clustalw/. The positions of TMhelices in both proteins was predicted using TMpred software http://bioinformatics.biol.uoa.gr/TMRPres2D/ and visualised using TMRPres2D java applet http://liao. cis.udel.edu/website/servers/TMMOD/.

\section{Additional material}

\begin{abstract}
Additional file 1: Additional Text. Section 1, Solexa genome resequencing; Section 2, Other mutations in AS-30CQ; Section 3, AS-15CQ and the origins of different haplotypes in subsequent clones; Section 4, Discontinuities in AJ allele frequency.

Additional file 2: (Table) Solexa whole-genome re-sequencing metrics.

Additional file 3: (Figure) LGS-pyro v LGS-Illumina. Comparison of genome scans (LGS-pyro (top), LGS-Illumina (bottom)) show near perfect correspondence between the two methodologies. Vertical axis (linear) indicates proportion of AJ alleles in parasites surviving $3 \mathrm{mg} \mathrm{CQ} \mathrm{kg}^{-1}$ day 1. Horizontal axis indicates chromosome number, top and bottom or genome co-ordinate (Kbase), top only. Position of mutations (AS-30CQ relative to AS-sens) are indicated at bottom of bottom panel (7 SNPS $\mathbf{x}, 2$ deletions $\cdot$ ).

Additional file 4: (Table) AS-30CQ Genome re-sequencing. Summary of all the mutations proposed in clone AS-30CQ (Additional File 1). Highlighted are mutations confirmed by di-deoxy sequencing (green), rejected mutations (red), a high confidence deletion (yellow) and low confidence mutations (orange). Read depth according to SSAHA2 is provided for SNPS. All quality scores for SNPs were according to SSAHA2. Small indel quality scores indicate the number of reads calling an indel divided by the total number of reads covering the indel. For large indels and CNVs, a comparative coverage was calculated as described (Methods section and Additional File 1).
\end{abstract}

Additional file 5: (Table) Genome-wide analysis of \% of bases with read-coverage $\geq 10$.

Additional file 6: (Figure) The appearance of mutations in the AS lineage. Mutations are described by chromosomal location, gene ID, specific amino acid change etc. Some were previously described [29,30] (blue). Novel mutations are identified here (red). For both PCHAS_030137 and ubp 1, alternative mutations arising between AS-3CQ and AS-15CQ (and individually selected in AS-15MF [31,35] and AS-ATN [55] during mefloquine and artesunate selection, respectively) are defined $(a-d)$. Refer to Additional File 1(section 3) for further details.

Additional file 7: (Figure) Chromosome $\mathbf{7}$ - $\mathbf{3 4}$ bp deletion. Close to the 3 ' end of gene PCHAS_072420, an alignment of nucleotide sequences for reference genome sequence (AS-WTSI), the sensitive AS lineage progenitor (AS-sens) and the drug resistant mutants AS-PYR, AS3CQ, AS-30CQ and AS-ART is shown. Symbols: -,34 bp deletion in AS-PYR and subsequent clones (wrt nt 197 - 230 (AS-WTSI arbitrary numbering) inclusive), 15 bp deletion in AS strains (wrt nt 306 - 320 (AJ arbitrary numbering) inclusive); ${ }^{*}$, nucleotides identical in all clones and strains 
investigated here (note high frequency of AS/AJ SNPS). 3' end of coding sequence of gene PCHAS_072420 is indicated (upper case, green highlighting); intron (lower case, grey highlighting), 3'-UT and intergenic region indicated (lower case); termination codon (red). Repetitive sequences which may mediate the $34 \mathrm{bp}$ and $15 \mathrm{bp}$ deletions are indicated (yellow) in individual representative clones.

Additional file 8: (Table) Discontinuity co-ordinates. Co-ordinates represent nucleotide position on chromosome relative to the AS-WTXI sequence assembly (Sanger Sept 2009); nd, cannot be determined.

Additional file 9: (Table) Primers used. These oligonucleotide primers were used to confirm the predicted mutations in AS lineage. Pairs of primers marked with * were also used for proportional sequencing.

\section{Abbreviations}

AAT1: amino acid transporter 1; ABC: ATP-binding cassette; CQ: chloroquine; CQ-R: chloroquine resistant: chloroquine resistance; CQ-hiR: high level chloroquine resistance; CRT: chloroquine resistance transporter; DV: digestive vacuole; LGS: linkage group selection; LGS-pyro: LGS analysed by pyrosequencing; LGS-Illumina: LGS analysed by quantitative Illumina wholegenome sequencing; Pgh-1: P-glycoprotein homologue-1; QTL: quantitative trait loci; SNP: single nucleotide polymorphism; TM: transmembrane; WGS: whole genome (re-)sequencing; WTSI: Wellcome Trust Sanger Institute.

\section{Acknowledgements}

The research was supported by Medical Research council (MRC), UK (G0400476). For personal support, we thank the Darwin Trust (KM), the MRC (AC, PH), BBSRC and EPSRC (LL), Fundação para a Ciencia e a Tecnologia, Portugal (AM, SB, LR), IHMT, Universidade Nova de Lisboa (PC), the School of Biological Sciences, MRC, UK (G0900740) (TM, MB), and the Wellcome Trust (RC, PH). The Centre for Immunity, Infection and Evolution is a Wellcome Trust Centre in Development (082611/Z/07/Z). The Centre for Systems Biology at Edinburgh is a Centre for Integrative Systems Biology (CISB) funded by BBSRC and EPSRC, reference BB/D019621/1. DNA sequencing was carried out in the GenePool Genomics Facility in the University of Edinburgh and we thank Marian Thomson, Karim Gharbi and other GenePool staff for assistance with sequencing. Members of the Pathogen Genomics group at the Wellcome Trust Sanger Institute and core sequencing and informatics groups gave valuable access to the $P$. chabaudi genome sequence, assembly and annotation data. We thank Richard Fawcett for technical assistance.

\section{Author details}

'Institute for Immunology and Infection Research, University of Edinburgh, Edinburgh, UK. ${ }^{2}$ Laboratory of Genetics and Wisconsin Institute for Discovery, University of Wisconsin-Madison, Madison, USA. ${ }^{3}$ The GenePool, University of Edinburgh, Edinburgh, UK. ${ }^{4}$ Centro de Malaria e Outras Doenças Tropicais/ IHMT/UEl Malaria, Lisbon, Portugal. ${ }^{5}$ Centro de Malaria e Outras Doenças Tropicais/IHMT/UEl Biologia Molecular, Lisbon, Portugal. ${ }^{6}$ Institute for Evolutionary Biology, University of Edinburgh, Edinburgh, UK. ${ }^{7}$ Centre for Immunity, Infection and Evolution, University of Edinburgh, Edinburgh, UK. ${ }^{8}$ Current address: The Wellcome Trust Sanger Institute, Hinxton, Cambridge, UK. ${ }^{9}$ Current address: Research Unit and Cardiology department, Funchal Hospital Center, Funchal. Madeira, Portugal. ${ }^{10}$ Current address: Microbiology, Molecular Genetics and Immunology, Kansas University Medical Center, Kansas City, USA. ${ }^{11}$ Current address: IPTSP, Universidade Federal de Goiás, Goiânia, Brasil.

\section{Authors' contributions}

$\mathrm{KM}, \mathrm{AC}, \mathrm{SB}$ and LR performed the biological and genetic experiments and confirmed mutations. LL, TC analysed and interpreted WGS SNP data in strain AJ, and quantitative LGS-Illumina data. AM analysed WGS for AS mutation detection. PC, MB, RC interpreted and supported research. PH conceived the study and managed the project. KM and PH wrote the paper with assistance from LL, TC and AM. All authors have read and approved the final manuscript.

Authors' information

The authors declare that they have no competing interests.
Received: 9 November 2011 Accepted: 21 March 2012

Published: 21 March 2012

\section{References}

1. Wellems TE, Walker-Jonah A, Panton LJ: Genetic mapping of the chloroquine-resistance locus on Plasmodium falciparum chromosome 7. Proc Natl Acad Sci USA 1991, 88(8):3382-3386.

2. Su X, Kirkman LA, Fujioka $H$, Wellems TE: Complex polymorphisms in an approximately $330 \mathrm{kDa}$ protein are linked to chloroquine-resistant $\mathrm{P}$. falciparum in Southeast Asia and Africa. Cell 1997, 91(5):593-603.

3. Fidock DA, Nomura T, Talley AK, Cooper RA, Dzekunov SM, Ferdig MT, Ursos LM, Sidhu AB, Naude B, Deitsch KW, et al: Mutations in the P. falciparum digestive vacuole transmembrane protein PfCRT and evidence for their role in chloroquine resistance. Mol Cell 2000, 6(4):861-871.

4. Durand R, Jafari S, Vauzelle J, Delabre JF, Jesic Z, Le Bras J: Analysis of pfert point mutations and chloroquine susceptibility in isolates of Plasmodium falciparum. Mol Biochem Parasitol 2001, 114(1):95-102.

5. Djimde A, Doumbo OK, Cortese JF, Kayentao K, Doumbo S, Diourte $Y$, Dicko A, Su XZ, Nomura T, Fidock DA, et al: A molecular marker for chloroquine-resistant falciparum malaria. N Engl J Med 2001, 344(4):257-263.

6. Sanchez CP, McLean JE, Stein W, Lanzer M: Evidence for a substrate specific and inhibitable drug efflux system in chloroquine resistant Plasmodium falciparum strains. Biochemistry 2004, 43(51):16365-16373.

7. Bray PG, Mungthin M, Hastings IM, Biagini GA, Saidu DK, Lakshmanan V, Johnson DJ, Hughes RH, Stocks PA, O'Neill PM, et al: PfCRT and the transvacuolar proton electrochemical gradient: regulating the access of chloroquine to ferriprotoporphyrin IX. Mol Microbiol 2006, 62(1):238-251.

8. Martin RE, Marchetti RV, Cowan Al, Howitt SM, Broer S, Kirk K: Chloroquine transport via the malaria parasite's chloroquine resistance transporter. Science 2009, 325(5948):1680-1682.

9. Fitch CD: Ferriprotoporphyrin IX, phospholipids, and the antimalarial actions of quinoline drugs. Life Sci 2004, 74(16):1957-1972.

10. Reed MB, Saliba KJ, Caruana SR, Kirk K, Cowman AF: Pgh1 modulates sensitivity and resistance to multiple antimalarials in Plasmodium falciparum. Nature 2000, 403(6772):906-909.

11. Sidhu $A B$, Valderramos $S G$, Fidock DA: pfmdr1 mutations contribute to quinine resistance and enhance mefloquine and artemisinin sensitivity in Plasmodium falciparum. Mol Microbiol 2005, 57(4):913-926.

12. Babiker HA, Pringle SJ, Abdel-Muhsin A, Mackinnon M, Hunt P, Walliker D: High-level chloroquine resistance in Sudanese isolates of Plasmodium falciparum is associated with mutations in the chloroquine resistance transporter gene pfcrt and the multidrug resistance Gene pfmdr1. J Infect Dis 2001, 183(10):1535-1538.

13. Duraisingh MT, von Seidlein LV, Jepson A, Jones $P$, Sambou I, Pinder M, Warhurst DC: Linkage disequilibrium between two chromosomally distinct loci associated with increased resistance to chloroquine in Plasmodium falciparum. Parasitology 2000, 121(Pt 1):1-7.

14. Valderramos SG, Valderramos JC, Musset L, Purcell LA, Mercereau-Puijalon O, Legrand E, Fidock DA: Identification of a mutant PfCRT-mediated chloroquine tolerance phenotype in Plasmodium falciparum. PLOS Pathog 2010, 6(5):e1000887.

15. Nomura T, Carlton JM, Baird JK, del Portillo HA, Fryauff DJ, Rathore D, Fidock DA, Su X, Collins WE, McCutchan TF, et al: Evidence for different mechanisms of chloroquine resistance in 2 Plasmodium species that cause human malaria. J Infect Dis 2001, 183(11):1653-1661.

16. Hunt P, Cravo PV, Donleavy P, Carlton JM, Walliker D: Chloroquine resistance in Plasmodium chabaudi: are chloroquine-resistance transporter (crt) and multi-drug resistance (mdr1) orthologues involved? Mol Biochem Parasitol 2004, 133(1):27-35.

17. Rosario VE: Genetics of chloroquine resistance in malaria parasites. Nature 1976, 261(5561):585-586.

18. Padua RA: Plasmodium chabaudi: genetics of resistance to chloroquine. Exp Parasitol 1981, 52(3):419-426.

19. Carlton J, Mackinnon M, Walliker D: A chloroquine resistance locus in the rodent malaria parasite Plasmodium chabaudi. Mol Biochem Parasitol 1998, 93(1):57-72.

20. Hunt P, Martinelli A, Fawcett R, Carlton J, Carter R, Walliker D: Gene synteny and chloroquine resistance in Plasmodium chabaudi. Mol Biochem Parasitol 2004, 136(2):157-164. 
21. Hayton K, Su XZ: Drug resistance and genetic mapping in Plasmodium falciparum. Curr Genet 2008, 54(5):223-239.

22. Ferdig MT, Cooper RA, MU J, Deng B, Joy DA, Su XZ, Wellems TE: Dissecting the loci of low-level quinine resistance in malaria parasites. Mol Microbiol 2004, 52(4):985-997.

23. Beez D, Sanchez CP, Stein WD, Lanzer M: Genetic predisposition favors the acquisition of stable artemisinin resistance in malaria parasites. Antimicrob Agents Chemother 2011, 55(1):50-55.

24. Mu J, Awadalla P, Duan J, McGee KM, Keebler J, Seydel K, McVean GA, Su XZ: Genome-wide variation and identification of vaccine targets in the Plasmodium falciparum genome. Nat Genet 2007, 39(1):126-130.

25. Van Tyne D, Park DJ, Schaffner SF, Neafsey DE, Angelino E, Cortese JF, Barnes KG, Rosen DM, Lukens AK, Daniels RF, et al: Identification and Functional Validation of the Novel Antimalarial Resistance Locus PF10_0355 in Plasmodium falciparum. PLoS Genet 2011, 7(4):e1001383.

26. Yuan J, Cheng KC, Johnson RL, Huang R, Pattaradilokrat S, Liu A, Guha R, Fidock DA, Inglese J, Wellems TE, et al: Chemical genomic profiling for antimalarial therapies, response signatures, and molecular targets. Science 2011, 333(6043):724-729.

27. Carter R, Hunt $P$, Cheesman S: Linkage Group Selection-a fast approach to the genetic analysis of malaria parasites. Int J Parasitol 2007, 37(34):285-293.

28. Hunt P, Afonso A, Creasey A, Culleton R, Sidhu AB, Logan J, Valderramos SG, McNae I, Cheesman S, do Rosario V, et al: Gene encoding a deubiquitinating enzyme is mutated in artesunate- and chloroquineresistant rodent malaria parasites. Mol Microbiol 2007, 65(1):27-40.

29. Hunt P, Martinelli A, Modrzynska K, Borges S, Creasey A, Rodrigues L, Beraldi D, Loewe L, Fawcett R, Kumar S, et al: Experimental evolution, genetic analysis and genome re-sequencing reveal the mutation conferring artemisinin resistance in an isogenic lineage of malaria parasites. BMC Genomics 2010, 11(1):499.

30. Martinelli A, Henriques G, Cravo P, Hunt P: Whole genome re-sequencing identifies a mutation in an $A B C$ transporter ( $m d r 2)$ in a Plasmodium chabaudi clone with altered susceptibility to antifolate drugs. Int $J$ Parasitol 2011, 41(2):165-171.

31. Borges S, Cravo P, Creasey A, Fawcett R, Modrzynska K, Rodrigues L, Martinelli A, Hunt P: Genomewide Scan Reveals Amplification of mdr1 as a Common Denominator of Resistance to Mefloquine, Lumefantrine, and Artemisinin in Plasmodium chabaudi Malaria Parasites. Antimicrob Agents Chemother 2011, 55(10):4858-4865.

32. Culleton R, Martinelli $A$, Hunt $P$, Carter R: Linkage group selection: rapid gene discovery in malaria parasites. Genome Res 2005, 15(1):92-97.

33. Cheesman S, Creasey A, Degnan K, Kooij T, Afonso A, Cravo P, Carter R, Hunt $P$ : Validation of Pyrosequencing for accurate and high throughput estimation of allele frequencies in malaria parasites. Mol Biochem Parasitol 2007, 152(2):213-219.

34. Grech K, Martinelli A, Pathirana S, Walliker D, Hunt P, Carter R: Numerous, robust genetic markers for Plasmodium chabaudi by the method of amplified fragment length polymorphism. Mol Biochem Parasitol 2002, 123(2):95-104

35. Cravo PV, Carlton JM, Hunt P, Bisoni L, Padua RA, Walliker D: Genetics of mefloquine resistance in the rodent malaria parasite Plasmodium chabaudi. Antimicrob Agents Chemother 2003, 47(2):709-718.

36. Martin RE, Kirk K: The malaria parasite's chloroquine resistance transporter is a member of the drug/metabolite transporter superfamily. Mol Biol Evol 2004, 21(10):1938-1949.

37. Jiang H, Patel JJ, Yi M, Mu J, Ding J, Stephens R, Cooper RA, Ferdig MT, Su XZ: Genome-wide compensatory changes accompany drug-selected mutations in the Plasmodium falciparum crt gene. PLoS One 2008, 3(6): e2484.

38. Warhurst D, Killick-Kendrick R: Spontaneous resistance to chloroquine in a strain of rodent malaria (Plasmodium berghei yoelii). Nature 1967, 213:1048-1049.

39. Janssen CS, Phillips RS, Turner CM, Barrett MP: Plasmodium interspersed repeats: the major multigene superfamily of malaria parasites. Nucleic Acids Res 2004, 32(19):5712-5720.

40. Cheng $Q$, Saul $A$ : The dihydrofolate reductase domain of rodent malarias: point mutations and pyrimethamine resistance. Mol Biochem Parasitol 1994, 65(2):361-363.
41. de Roode JC, Helinski ME, Anwar MA, Read AF: Dynamics of multiple infection and within-host competition in genetically diverse malaria infections. Am Nat 2005, 166(5):531-542.

42. de Roode JC, Pansini R, Cheesman SJ, Helinski ME, Huijben S, Wargo AR, Bell AS, Chan BH, Walliker D, Read AF: Virulence and competitive ability in genetically diverse malaria infections. Proc Natl Acad Sci USA 2005, 102(21):7624-7628.

43. Miki A, Tanabe K, Nakayama T, Kiryon C, Ohsawa K: Plasmodium chabaudi: association of reversal of chloroquine resistance with increased accumulation of chloroquine in resistant parasites. Exp Parasitol 1992, 74(2):134-142.

44. Ohsawa K, Tanabe K, Kimata I, Miki A: Ultrastructural changes associated with reversal of chloroquine resistance by verapamil in Plasmodium chabaudi. Parasitology 1991, 103(Pt 2):185-189.

45. Tanabe K, Kato M, Izumo A, Hagiwara A, Doi S: Plasmodium chabaudi: in vivo effects of $\mathrm{Ca} 2+$ antagonists on chloroquine-resistant and chloroquine-sensitive parasites. Exp Parasitol 1990, 70(4):419-426.

46. Ehrenreich IM, Torabi N, Jia Y, Kent J, Martis S, Shapiro JA, Gresham D, Caudy AA, Kruglyak L: Dissection of genetically complex traits with extremely large pools of yeast segregants. Nature 2010, 464(7291):1039-1042

47. Dharia NV, Sidhu AB, Cassera MB, Westenberger SJ, Bopp SE, Eastman RT, Plouffe D, Batalov S, Park DJ, Volkman SK, et al: Use of high-density tiling microarrays to identify mutations globally and elucidate mechanisms of drug resistance in Plasmodium falciparum. Genome Biol 2009, 10(2):R21.

48. Rottmann M, McNamara C, Yeung BK, Lee MC, Zou B, Russell B, Seitz P, Plouffe DM, Dharia NV, Tan J, et al: Spiroindolones, a potent compound class for the treatment of malaria. Science 2010, 329(5996):1175-1180.

49. Carter R, Walliker D: New observations on the malaria parasites of rodents of the Central African Republic - Plasmodium vinckei petteri subs. nov. and Plasmodium chabaudi Landau 1965. Annals of Tropical Medicine and Parasitology 1975, 69:187-196.

50. Walliker D, Carter R, Sanderson A: Genetic studies on Plasmodium chabaudi: recombination between enzyme markers. Parasitology 1975 70(1):19-24.

51. Li H, Ruan J, Durbin R: Mapping short DNA sequencing reads and calling variants using mapping quality scores. Genome Res 2008, 18(11):1851-1858.

52. Ning Z, Cox AJ, Mullikin JC: SSAHA: a fast search method for large DNA databases. Genome Res 2001, 11(10):1725-1729.

53. Li H, Durbin R: Fast and accurate short read alignment with BurrowsWheeler Transform. Bioinfomatics 2009, 25:1754-1760.

54. Hunt $P$, Fawcett $R$, Carter $R$, Walliker D: Estimating SNP proportions in populations of malaria parasites by sequencing: validation and applications. Mol Biochem Parasitol 2005, 143(2):173-182.

55. Afonso A, Hunt P, Cheesman S, Alves AC, Cunha CV, do Rosario V, Cravo P: Malaria parasites can develop stable resistance to artemisinin but lack mutations in candidate genes atp6 (encoding the sarcoplasmic and endoplasmic reticulum Ca2+ ATPase), tctp, mdr1, and cg10. Antimicrob Agents Chemother 2006, 50(2):480-489.

56. Carlton JM, Hayton K, Cravo PV, Walliker D: Of mice and malaria mutants: unravelling the genetics of drug resistance using rodent malaria models. Trends Parasitol 2001, 17(5):236-242.

doi:10.1186/1471-2164-13-106

Cite this article as: Kinga Modrzynska et al:: Quantitative genome resequencing defines multiple mutations conferring chloroquine resistance in rodent malaria. BMC Genomics 2012 13:106. 